\title{
Kritische Anmerkungen zum psychiatrischen Krankheitsverständnis
}

\author{
D. Hell
}

\begin{abstract}
Die moderne Psychiatrie geht davon aus, dass psychisches Erleben operationalisiert, d.h. objektiviert und gemessen werden kann. Sie beschreibt psychische Störungen als Symptommuster und versucht, dieses Symptommuster auf neuronale Prozesse zurückzuführen. Dieses Vorgehen setzt implizit voraus, dass psychische Phänomene in gleicher Weise wie beobachtbare Verhaltensweisen übereinstimmend festgehalten und untersucht werden können.
\end{abstract}

Eine solche Annahme ist aber Gegenstand kontroverser wissenschaftstheoretischer Diskussionen und kann empirisch nicht belegt werden. Es sind viele komplexe Erlebnisse vorstellbar, die so intim sind, dass sie nicht mit objektiven Methoden erfasst werden können. Was eine Person «aus erster Hand» erlebt, ist zudem nicht einfach in eine Aussenperspektive übertragbar.

Korrespondenz:

Prof. Dr. med. Daniel Hell Psychiatrische Universitätsklinik

Lenggstrasse 31

CH-8029 Zürich

\section{Psychisch krank - ein Konstrukt?}

Die Psychiatrie ist eine praktische Wissenschaft, die sich wenig mit erkenntnistheoretischen Grundfragen auseinandersetzt. Dies kann sich rächen, wenn aus undurchdachten A-prioriAnnahmen schwerwiegende soziale Folgerungen gezogen werden. Ein historisches Beispiel stellt die Eugenik dar.

Gerade in sozialen Umbruchzeiten ist die Psychiatrie gefordert. Dann kann die Not und Verunsicherung in der Bevölkerung derart anwachsen, dass der Ruf nach einfachen technischen Lösungen für komplexe menschliche Probleme immer grösser wird. Setzt man sich mit den grundlegenden Fragen seiner Disziplin nicht auseinander, droht, was Karl Jaspers in folgende Worte gefasst hat: «Wer meint, die Philosophie ausschalten und sie als belanglos beiseite lassen zu können, wird von ihr in ungeklärter Gestalt überwältigt.» In andere Worte gefasst: Wer sich den grundlegenden theoretischen Problemen nicht stellt, wird von ihnen eingeholt und überwältigt. Karl Jaspers war der Auffassung, dass sich jeder Arzt nach einer bestimmten Weltsicht verhalte, ob er darum wisse oder nicht. Deshalb besteht die Wahl nicht darin, eine Vorstellung eine Philosophie - über den Menschen zu haben oder auf eine solche zu verzichten. Der Arzt habe nur die Wahl, sich dieser Konzeption bewusst zu sein oder nicht.

\section{Geschichtlicher Hintergrund der Psychiatrie}

Die Heilkunde (Iatreia) der Seele (Psyche) ist ein Kind der Aufklärung. In der Zeit der Aufklärung wurden Gefühle im Anschluss an Descartes nicht mehr als Ausdruck einer immateriellen Seele verstanden, sondern als ein (an den Körper gebundenes) Phänomen, das dem Studium des menschlichen Geistes zugänglich ist. Damit konnte sich auch die Überzeugung durchsetzen, dass seelisches Erleben erkranken kann. Eine solche Vorstellung war dem Denken vor der Aufklärung fremd. Ging es früher um das Heil der Seele - war also Seelsorge gefragt -, verbreitete sich jetzt die Vorstellung einer Erkrankungsmöglichkeit der Seele - und damit die Forderung nach der Behandlung seelischer Störungen. Zum Erfolg dieses Paradigmenwechsels trugen neben medizinischen Fortschritten auch eingreifende gesellschaftliche und sozioökonomische Veränderungen bei. So konnte unmittelbar nach der bürgerlichen Revolution in Frankreich durchgesetzt werden, dass sogenannte Irre - als psychisch Kranke neu definiert - nicht mehr mit anderen Auffälligen und Ordnungsstörern (wie Vagabunden, Rechtsbrechern und Prostituierten) in Internierungshäusern zusammen eingesperrt, sondern in separaten medizinischen Abteilungen behandelt wurden.

Psychiatriegeschichtlich ist Philippe Pinel besonders wichtig. Er übernahm als Arzt in Paris 1793 und 1795 die Leitung der gefängnisartig geführten Internierungshäuser Bicêtre (für Männer) und Salpêtrière (für Frauen). Nach kurzer Zeit gelang es ihm, viele Zwangsmittel wie Ketten, Hals- und Fussringe abzuschaffen und neue Behandlungsweisen einzuführen.

Pinel verkörpert den geschichtlichen Wendepunkt, an dem der psychisch Auffällige und Leidende vom Störenden, Besessenen oder Unvernünftigen zum Kranken wurde. Pinel machte ihn zum medizinischen Fall - und zwar in grosser Zahl. Er entwickelte früh einen Typus der Anstaltspsychiatrie, welcher die systematische Beobachtung und Untersuchung der Patientinnen 
und Patienten in den Vordergrund stellte. Durch seine exakten Beschreibungen der Manie, Melancholie, Demenz und des Schwachsinns etablierte sich das klinische Fach der Psychiatrie als Spezialgebiet der Medizin. Als Basis dienten Pinel die Krankengeschichten. Das von ihm und seinen Schülern geforderte Anstaltswesen verbreitete sich - auch dank gesetzlicher Unterstützung - mit immer neuen Institutionsgründungen bald über ganz Frankreich, so dass um 1850 fast jedes französische Departement eine eigene Irrenanstalt hatte.

Eine ähnliche Verbreitung von Irrenhäusern fand mit einer gewissen zeitlichen Verzögerung auch in andern europäischen Ländern und in Nordamerika statt.

Die neu erstandene Heilkunde der Seele hatte aber bald mit überwunden geglaubten Problemen zu kämpfen. So folgte der Abschaffung von eisernen Ketten und Ringen bald die Einführung von Zwangsstuhl, Zwangsjacke und Gummizelle. Die Zwangsmittel wurden raffinierter, dienten aber dem gleichen Zweck. Auch die Denkansätze und Gedankengänge der Psychiatrie wurden dem neuen Paradigma angepasst, ohne sich aber ganz von den früher herrschenden Grundsätzen zu lösen. So wurde manches, was vorher sündig war, jetzt als krank erklärt. Was früher störte und zur gesellschaftlichen Ächtung führte, wurde jetzt als «psychopathisch» oder «irr» nicht viel weniger abgelehnt. Wer früher arm und hilflos war und sich dieses Schicksal nach öffentlicher Meinung selber oder den Vorfahren zuzuschreiben hatte, litt nun nach der verbreiteten Lehre von Morel an einer fortschreitenden Degeneration der Nerven.

Mit diesem überzeichneten Abriss der psychiatrischen Entwicklung im 19. Jahrhundert möchte ich deutlich machen, dass sich die Definition von seelischer Krankheit von Anfang an sehr stark nach den sozialen Wertvorstellungen ausrichtete. Auch ein Jahrhundert nach dem berühmten Satz von Wilhelm Griesinger (1817-1869) «Geisteskrankheiten sind Gehirnkrankheiten» wurden seelische Krankheiten im wesentlichen nicht aufgrund neuronaler Befunde, sondern aufgrund kultureller und gesellschaftlicher Normvorstellungen definiert und diagnostiziert. Es ist wohl kein Zufall, dass auch moderne epidemiologische Studien nachweisen, dass schwer psychisch Kranke überwiegend arm und gesellschaftlich schlecht positioniert sind (was für viele somatische Erkrankungen wie z.B. Herzkrankheiten nicht zutrifft).

\section{Krankheit als gestörte Ordnung}

Es wirkt sich bis heute aus, dass die Entwicklung psychiatrischer Krankheitskonzepte hauptsächlich an Menschen erfolgte, die zuerst gesellschaftlich als abnorm ausgegrenzt und zwangsweise in geschlossenen Anstalten interniert wurden. Solche Patienten bildeten auch den Erfahrungshintergrund von Emil Kraepelin (1856-1926), der die noch heute geltende Krankheitseinteilung psychischer Störungen massgeblich beeinflusst hat. Historisch wurde die Verbindung von Ausgrenzungsprozessen und diagnostischen Beurteilungen innerhalb der Psychiatrie lange Zeit zuwenig wahrgenommen, weil die sozialen Normvorstellungen den biologischen Normvorstellungen gleichgesetzt wurden. So wurde die im 19. Jahrhundert aufkommende physiologische Vorstellung eines inneren Gleichgewichtes («milieu intérieur») auf soziale Ordnungsprinzipien übertragen. Dem geordneten «milieu intérieur» entsprach eine geordnete Sozialordnung. Dank dieses «naturalistischen Fehlschlusses» war es möglich, dass sich zwei verschiedene Normsysteme - das gesellschaftliche und das biologisch-physiologische - in unsäglicher Weise miteinander verbinden konnten. Die biologisch-physiologische Ordnung wurde auf den ganzen Menschen und schliesslich auch auf die soziale Ordnung extrapoliert. Die naturwissenschaftlichen Gesetze wurden zur Begründung bestimmter gesellschaftlicher Wertvorstellungen eingesetzt. Umgekehrt wurden soziale Normen herangezogen, um das physiologische Geschehen zu illustrieren.

Diese Vermischung von biologischen und gesellschaftlichen Ordnungskriterien entzündete sich vor und nach dem 1 . Weltkrieg zum Flächenbrand, als soziales Elend um sich griff. Je grösser die soziale Not wurde, um so mehr wurde nach einer einfachen technischen Lösung gesucht, die in vermeintlich voraussagbarer und kontrollierter Weise das Elend beseitigen könnte. Genau eine solche rationale und effiziente Lösung versprach die Eugenik, die sich unter dem Einfluss von Sozialdarwinismus und psychiatrischer Degenerationslehre gebildet hatte.

\section{Neue Definitionen von psychisch krank im 20. Jahrhundert}

Nach den Katastrophen der ersten Hälfte des 20. Jahrhunderts veränderte sich die Situation. Das starre Ordnungsdenken wurde durch eingreifende Kriegserfahrungen und soziale Umwälzungen erschüttert. Gleichzeitig rückte die 
Fähigkeit, schwierige Lebenssituationen durch ein entsprechendes Anpassungsvermögen zu meistern, in den Vordergrund. Unter den veränderten gesellschaftlichen Bedingungen wandelte sich auch das Verständnis psychischer Krankheiten. Das Kriterium einer instabilen Ordnung wurde zunehmend durch das Kriterium einer unflexiblen Anpassung ersetzt. Neurotische Anpassungsstörungen wurden zur häufigsten Diagnose. Sie wurden unter dem Einfluss der aufkommenden psychoanalytischen Lehre als Ausdruck innerer Konflikte gesehen, welche sich hinderlich auf die sozialen Beziehungen eines Menschen auswirken. Die klassische Verhaltenslehre ging noch einen Schritt weiter und setzte psychische Störungen mit inadäquatem Verhalten gegenüber der Umwelt gleich.

In den letzten Jahrzehnten haben sich die Grundlagen der psychiatrischen Krankheitslehre nochmals verändert. Anstelle von Ordnung oder Anpassung entscheidet immer mehr das Befinden der Menschen über Krankheit oder Gesundheit. Nach der aktuellen Definition der Weltgesundheitsbehörde ist ein Mensch krank, wenn sein Wohlbefinden gestört ist. Konsequenterweise werden Befindlichkeitsstörungen, die früher als Normvariante angesehen wurden wie Verstimmungszustände vor der Menstruation oder extreme Schüchternheit (soziale Phobie) -, zu behandlungsbedürftigen Störungen. Demgegenüber wurden früher als krank (weil ordnungswidrig oder bezüglich Fortpflanzung dysfunktionell) eingeschätzte Verhaltensweisen wie die Homosexualität als potentiell lustvoll und damit als gesund betrachtet.

\section{Sozialgeschichte und Psychiatrie}

So sehr gesellschaftliche Verhältnisse die psychiatrische Krankheitslehre geprägt haben, so sehr gilt es auch, den umgekehrten Vorgang in Betracht zu ziehen, nämlich die Auswirkung des psychiatrischen Verständnisses auf die gesundheitspolitische Praxis und auf das Recht.

Mittlerweile hat sich eine ganze Reihe von Wissenschaftsdisziplinen (in Philosophie, Soziologie und Geschichte) entwickelt, die dem $\mathrm{Zu}$ sammenhang zwischen Sozialgeschichte einerseits und wissenschaftlichen Vorstellungen anderseits nachgehen. Sie haben deutlich gemacht, dass die Suche nach Naturgesetzen in der Medizin keineswegs von sozialen Wertvorstellungen unabhängig ist. Das Experiment holt nicht einfach «Wissen» aus dem Körper heraus, sondern verwandelt im Grunde den Körper in einen Text, in ein «lebendes Dossier», das nach einer be- stimmten, kulturell vermittelten Weise gelesen wird. Mit dem Studium des «lebenden Dossiers» wandelt sich auch das bisherige Wissen. Dabei kann der zu lesende Text immer komplizierter werden, so dass sich der ursprüngliche Körper in abstrakte Vorstellungen verflüchtigt.

So hat die Physiologie im letzten Jahrhundert den Körper «verflüssigt» und «elektrisiert», indem sie die Regelsysteme der Hormone und der Nerven herausgearbeitet hat. Heute wird der Körper durch die Molekularbiologie zum Ort der Informationsvermittlung. Der Text, nach dem im Körper gesucht wird, ist derart abstrakt geworden, dass der wirkliche Körper, der betastet und gespürt werden kann, im wissenschaftlich geprägten Alltag verlorenzugehen droht.

\section{Der verrechnete Mensch}

Damit stellt sich der modernen Medizin eine besondere Herausforderung, mit der ich mich im eben vorgelegten Buch «Seelenhunger - der fühlende Mensch und die Wissenschaft vom Leben» auseinandersetze. Wie kann man dem Leib eines Menschen - seinem Empfinden und Fühlen - gerecht werden, wenn der medizinalisierte Mensch im Zeitalter der Evidence-based Medicine immer mehr einer «Datenbank» oder einer «Informationshülle» zu ähneln beginnt? Wie kann die Medizin den «inneren» Erlebensraum der Menschen, der sich in der Geschichte des Abendlandes symbolhaft als Seelenvorstellung ausdifferenziert hat, in Zukunft in ihre postmodernen Konzeptionen integrieren?

Die Möglichkeit, mit immer mehr Informationen die strukturellen Zusammenhänge eines Menschen zu charakterisieren - Stichwort: der gläserne Mensch -, geht mit dem psychiatrischen Versuch einher, auch die Persönlichkeitseigenschaften und die psychischen Problemstellungen eines Menschen in einzelne Informationseinheiten aufzulösen. Eine solche Tendenz führt leicht dazu, dass die Wissenschaft der Psychiatrie das Interesse an der Geschichte eines Menschen verliert, also an dem, was ein Mensch über sich selber zu erzählen weiss und wie er seine Erfahrungen zu einer zusammenhängenden, sinnmachenden Geschichte verknüpft. Die akademische Psychiatrie konzentriert sich denn auch schon heute verstärkt auf abfragbare Eigenschaften, die als Informationen digital verrechnet werden können.

Solche abfragbaren Eigenschaften betreffen z.B. die Bewusstseinslage (ob ein Mensch hellwach oder schläfrig ist), das Gedächtnis (ob er über eine gute oder schlechte Merkfähigkeit bzw. 
über ein erhaltenes oder beeinträchtigtes Altgedächtnis verfügt), das formale Denken (ob ein ungestörter Gedankenfluss vorliegt oder eine Denkhemmung besteht) oder die Stimmung (ob sie gehoben, ausgeglichen oder niedergeschlagen ist). Durch ein Zusammenstellen solcher Symptome zu einem Symptommuster entsteht ein neues «Bild» vom Menschen.

Dieses aus Informationseinheiten zusammengesetzte und mit Computerhilfe errechnete «Bild» eines Menschen ist ein «Roboterbild». Es unterscheidet sich vom Gesamteindruck eines Menschen, den ich aufgrund einer Begegnung (seiner Erscheinung, seiner geäusserten Vorstellungen und seiner Kommunikationsweise) gewinne. Das digitalisierte Bild hat keine wirkliche Gestalt, enthält keine persönliche Botschaft und keinen Sinn. Es ist reines Faktum, digitale Verrechnung unzähliger Daten. Es erhält erst Gestalt, Botschaft und Sinn, wenn ein Beobachter dieses «Roboterbild» betrachtet und sich darunter aufgrund seiner eigenen Erfahrungen ein «analoges Bild» macht. Allerdings kann diese sinnstiftende Fähigkeit eines Beobachters darüber hinwegtäuschen, dass der digitale Abdruck künstlich ist und nicht auf selbergemachten Erfahrungen basiert. Um solche Erfahrungen zu gewinnen, wäre ein Dialog mit dem zu beurteilenden Menschen nötig, der über die digitale Erfassung einzelner Eigenschaften hinausgreift.

Natürlich liegt die Problematik der aktuellen Diagnostik nicht in der computergestützten Vermessung von menschlichen Eigenschaften, sondern in der unkritischen Annahme, eine solche Methode könne den Dialog mit einer hilfesuchenden Person, bzw. das Verständnis für ihre aktuelle Lage und ihre Geschichte, ersetzen. Werden intuitiv erfasste Eindrücke, die in persönlichen Auseinandersetzungen zustande kommen, mit einer solchen Methode punktuell überprüft, ist dagegen nicht nur nichts einzuwenden. Ein solches Vorgehen verbessert vielmehr die individuelle Beurteilung.

\section{Apersonale Diagnostik?}

Unter amerikanischer Federführung setzt sich jedoch in der modernen Psychiatrie seit 1980 mit Einführung des diagnostischen und statistischen Manuals Mentaler Störung (DSM III) - eine Entwicklung durch, welche die psychiatrische Diagnostik auf abfragbare Merkmale oder Symptome abstützt, ohne zu fordern, dass die Angaben der Patientinnen und Patienten in einen $\mathrm{Zu}$ sammenhang mit ihrer persönlichen Geschichte und den aktuellen Lebensumständen gebracht werden. Dieses symptomorientierte Vorgehen lässt die Diagnostik leichter handhaben. Zudem erfüllt sie Forschungsbedürfnisse, da auf diese Weise eine gute diagnostische Übereinstimmung zwischen verschiedenen Untersuchern erreicht werden kann. Sie verleitet aber auch zum Trugschluss, mit objektiven Beobachtungen das subjektive Erleben einfangen zu können.

So wird zunehmend häufiger davon ausgegangen, dass bestimmte mit Fragebogen erfasste Eigenschaften eines Menschen einer spezifischen Veränderung des Gehirns entsprechen würden. Bei einem solchen Vorgehen wird aus der grundsätzlich unbestrittenen Annahme, dass alles Erleben und Verhalten eine körperliche Grundlage hat, die unerlaubte Schlussfolgerung gezogen, eine psychologisch definierte Problematik, z.B. ein depressives Symptommuster, müsse notwendigerweise auch eine gleichartige Veränderung des Stoffwechsels oder der neuronalen Aktivität ausdrücken.

Oder bildlich formuliert: Es wird angenommen, die psychosoziale Definition der Störung erfasse die Trennung von körperlichen Funktionseinheiten an ihren «Nahtstellen». In Tat und Wahrheit kann aber eine bestimmte Symptomkonstellation (wie Niedergeschlagenheit, Ermüdbarkeit, Unschlüssigkeit, Appetit- und Einschlafstörung als depressives Symptommuster) durch verschiedenartige körperliche Zustände abgebildet werden und muss kein einheitliches neuronales Aktivitätsmuster widerspiegeln.

Die Annahme einer Identität von psychosozialem Symptommuster und körperlicher Funktionsstörung beruht eher auf der Suggestivkraft der Sprache als auf rational begründeten Zusammenhängen.

\section{Schlussfolgerung}

Was als krank bezeichnet wird, ist von kulturellen Bedingungen abhängig. Die Kriterien für Gesundheit und Krankheit haben sich nicht nur über grosse Zeitepochen hinweg, sondern auch im Verlaufe des letzten Jahrhunderts grundlegend verändert. Die modernen psychiatrischen Diagnosen sind Kurzbeschreibungen. Sie fassen einzelne Symptome in ihrem zeitlichen Auftreten zusammen. Sie sind nützlich für die Kommunikation zwischen Spezialistinnen und Spezialisten. Sie können statistische Hinweise geben, welche Behandlungsweise einer anderen Behandlungsform im Gruppenvergleich kurzbis mittelfristig überlegen ist. Ein weiterer Vorteil der modernen Diagnostik liegt darin, dass sie anders als ältere Einteilungsversuche keine 
ungesicherten Hypothesen über Krankheitsursachen und Prognosen enthalten.

Der Vorteil ihrer expliziten Bescheidenheit und Einfachheit wird jedoch zum gravierenden Nachteil, wenn den modernen Krankheitsbegriffen eine Aussagekraft zugemutet wird, die sie nicht erfüllen können. So sind auch die modernen Krankheitsbegriffe nicht geeignet, die individuelle Problematik eines Menschen zu erfassen. Ihre Objektivität geht auf Kosten all dessen, was ein Individuum ausmacht: wie eine Person mit Belastungen umgeht, was diese Person als negativ oder positiv bewertet, inwieweit sie Leiden zu ertragen bereit ist, wie sie zum Krankheitsverständnis der angewandten Diagnostik steht und welche Behandlungsform ihr entgegenkommt.

Aufgrund der in diesem Artikel dargestellten Beobachtungen aus der Psychiatriegeschichte lassen sich bestimmte Risiken für die Zukunft ableiten. Eine unkritische Anwendung des modernen Krankheitskonzepts birgt die Gefahr in sich, Patientinnen und Patienten einem neuen und wenig durchdachten gesellschaftlichen Normsystem zu unterwerfen. Denn auch Krankheitskriterien, die primär auf einer Störung des Wohlbefindens beruhen, können den einzelnen einer gesundheitspolitischen Einordnung unterwerfen, die weitreichende Folgen hat. Zwar dient die statistische Normierung psychischer Auffälligkeiten im neuen System nicht mehr ordnungspolitischen Zwecken wie zur Gründungszeit der Psychiatrie, doch hat sie eine finanzpolitische Funktion. Die neuen (sogenannten atheoretischen) Diagnosekriterien können unter dem Vorwand, auf repräsentative Weise psychisch kranke Menschen in der Bevölkerung zu charakterisieren, dazu verwendet werden, eine Selektion des Leidens durchzuführen. Daraus dürfte nicht wie früher ein Ausgrenzungs- und Internierungsdruck entstehen, sondern ein Anpassungsund Behandlungsdruck, bestimmte Schwierigkeiten mit gesellschaftlich sanktionierten Methoden zu therapieren oder sonst auf finanzielle Unterstützung verzichten zu müssen.

Schon deshalb ist es so wichtig, sich bewusst zu sein, dass Krankheitsbeschreibungen immer Konventionen darstellen und dass die Zuordnung eines Krankheitsbegriffes $\mathrm{zu}$ einer bestimmten Person nicht das betroffene Individuum als Ganzes charakterisieren kann.

Eine ausführlichere Darstellung und detaillierte Literaturangaben finden sich in Hell D. Seelenhunger - der fühlende Mensch und die Wissenschaften vom Leben. Bern: Huber; 2002; speziell im 3. Kapitel «Kranke Seele? - Zur Geschichte der Psychiatrie». 